\title{
DISEÑAR UN PROYECTO (20 PASOS INICIALES...)
}

\author{
Carlos Alberto Caudana
}

...hasta el viaje más largo comienza por el primer paso

Proverbio chino

El pensamiento humano posee "un aspecto realizativo, concretamente comprometido con las materias $y$ con las técnicas [...] y un aspecto procesual y tentativo, típico de un hacer que mientras hace inventa el modo de hacer"

Luigi Pareyson

Estética 
Los orígenes de la investigación científica se remontan e incluso superponen con los procesos mismos de conformación y desarrollo intelectual del hombre, comprometiéndose desde su propio nacimiento con la problemática supervivencia de la especie humana.

Las actividades investigativas implican el desenvolvimiento de tareas metódicas, ordenadas, que a la vez avancen y/o rectifiquen (/o-nuevo-conocido) a partir de experiencias anteriores, sean éstas remotas o recientes. Es por ello que (aunque dicho en términos muy generales) en toda investigación científica pueden distinguirse algunas instancias claves y determinantes'; por ejemplo:

$>$ el planteamiento y formulación de un problema, duda o dificultad que inquieta al investigador (y con él, a toda la comunidad científica y a la misma sociedad), como primera cuestión a resolver;

> la etapa propedéutica de la indagación, o sea de preparación de todo lo necesario para resolver dicho problema (tareas previas, cuestiones técnico-materiales que permitan llevar a cabo la investigación, etc.);

$>$ la instancia heurística de la pesquisa, o de búsqueda sistemática de los materiales o elementos requeridos (bibliografía complementaria ${ }^{2}$, eventuales fuentes documentales sobre el tema investigado, etc.) para resolverlo adecuadamente; $>$ el momento hermenéutico de la investigación, en que se analizan e interpretan (críticamente) los datos, conocimientos e información indagada en las etapas previas, a fin de resolver práctica y/o teóricamente, aunque sea en forma transitoria, el problema en cuestión ${ }^{3}$;

\footnotetext{
1 Estos "momentos modulares" del proceso de investigación científica constituyen etapas lógicas más que cronológicas (aunque, en la mayoría de los casos, el ordenamiento temporal de las acciones investigativas se corresponde con el desarrollo lógico de los procesos y las actividades del investigador).

${ }^{2}$ Como suele decirse: el desarrollo de una investigación científica no consiste sólo en una investigación bibliográfica, pero no puede ocurrir ni avanzar sin ella.

${ }^{3}$ El momento de la reformulación y contrastación de las hipótesis de investigación se corresponde con esta etapa hermenéutica o de análisis, lectura e interpretación crítica de los datos, informaciones, etc.
} 
> la difusión, publicación, etc. de los resultados del trabajo de investigación, es decir la instancia de confrontación de lo producido con la opinión de los demás, incluso en ámbitos ajenos a la especialidad, cerrando así el ciclo de las pesquisas y evaluaciones críticas realizadas ${ }^{4}$.

\section{ABORDAR LA CUESTIÓN}

Proceso de investigación y diseño de investigación son fases interactuantes de una misma problemática ${ }^{5}$. En el momento inicial de toda investigación es posible diferenciar dos grandes etapas:

$>$ una instancia reflexiva, preliminar, comprometida con la propia formación e idiosincrasia del investigador (con sus conocimientos y experiencias sobre los fenómenos por investigar y, también, con sus opciones éticas e ideológicas), en la que se procurarán establecer los marcos referenciales desde los que partirá el subsiguiente proceso de investigación;

$>$ una etapa de diseño o de planificación de las actuaciones que se ejecutarán en las fases posteriores de la investigación en curso.

En tal sentido, las actividades correspondientes al diseño de investigación suelen estructurarse a partir de cuestiones como las siguientes: (1) ¿qué tipo de diseño resulta más adecuado a la formación, experiencia e idiosincrasia del investigador?; (2) ¿qué o quién va a ser estudiado?; (3) ¿qué método de indagación se va a utilizar?; (4) ¿qué técnicas se emplearán para recoger, analizar e interpretar los datos?; (5) ¿desde qué perspectiva o marco teórico-conceptual se elaborarán las conclusiones de la investigación?; etc., etc.

\footnotetext{
${ }^{4}$ Cualquiera sea su nivel de formulación y estado de formalización, lo importante del desarrollo de una investigación científica es asegurar su adecuada difusión y discusión: en ello radica, precisamente, la índole y razón de ser del "pensamiento" y del "hacer" de la ciencia.

${ }^{5}$ Si consideramos al diseño de investigación en su amplia acepción de planificación de actividades (destinadas a solucionar los problemas o bien a contestar las preguntas planteadas), puede entonces ser entendido como un eslabón o puente entre las cuestiones de investigación y las diferentes soluciones propuestas.
} 
"La elección paradigmática que se haya realizado en la etapa anterior determinará en gran medida el diseño de la investigación. Desde la rigurosidad extrema, característica del paradigma positivista o postpositivista, hasta el carácter emergente propiciado por los paradigmas basados en la teoría crítica o el constructivismo" (G. Rodríguez Gómez y otros,1999:67).

A continuación propongo un conjunto de interrogantes iniciales y consignas básicas más o menos "instrumentales", en cualquier caso relacionadas con prácticas introductorias al hacer (y a la reflexión sobre el hacer) investigativo ${ }^{6}$ en determinados espacios del conocimiento interdisciplinar.

Más precisamente: estas cuestiones y actividades sugeridas se vinculan con determinadas prácticas y enfoques característicos de la (denominada) investigación cualitativa ${ }^{7}$, y se aplican a la resolución de problemas en el campo de las humanidades y las ciencias sociales, u otros dominios aún más específicos. Por ejemplo: el de las teorías y metodologías semióticas, o el del análisis e interpretación de los discursos artísticos, audiovisuales, mediáticos, espectaculares, etc.

\footnotetext{
${ }^{6}$ Algunas lecturas iniciales sugeridas, para los interesados en introducirse o profundizar en estas cuestiones: (1) Eco, U. [1995]: Cómo se hace una tesis. Barcelona: Gedisa. (2) Giacobbe, M. y Moscoloni, N. [1999]: Aprender investigando ciencias sociales. Rosario: Cerider. (3) Hernández Sampieri, R. y otros [1997]: Metodología de la investigación. México: McGraw-Hill. (4) Mancuso, H. [1999]: Metodología de la investigación en ciencias sociales. Lineamientos teóricos y prácticos de semioepistemología. Buenos Aires: Paidós. (5) Vasilachis, I. y otros [1993]: Métodos cualitativos, I ("Los problemas teórico-epistemológicos") y II ("La práctica de la investigación"). Buenos Aires: Ceal.

${ }^{7}$ La investigación cualitativa supone la utilización de diversos materiales (entrevistas, experiencias personales, historias de vida, observación de imágenes, etc.) que permiten describir las situaciones problemáticas e interpretar los significados que adquieren para las personas implicadas. En términos de Denzin y Lincoln [cf. Handbook of Qualitative Research. Londres: Sage], constituye "un campo interdisciplinar, transdisciplinar y en muchas ocasiones contradisciplinar"; "multiparadigmático" y "multimetódico" en su enfoque, y quienes la practican se someten "a la perspectiva naturalista y a la comprensión interpretativa de la experiencia humana" [1994: 576]. (Para un estudio introductorio a la naturaleza y principales enfoques, métodos y prácticas de dichas indagaciones, se recomienda la lectura de Metodología de la investigación cualitativa de G.Rodríguez Gómez y otros autores [Málaga: Eds. Aljibe - $2^{\mathrm{a}}$ ed. 1999]).
} 
La mayoría de estas consignas fueron inicialmente pensadas ${ }^{8}$ como un modo relativamente "práctico" de orientar a los cursantes de grado e investigadores en formación, respecto del abordaje de actividades y requerimientos puntuales de algunas Cátedras y Seminarios de nuestro Proyecto y del Área Curricular de Semiótica, que se dictan o desarrollan en la Facultad de Humanidades y Ciencias de la Universidad Nacional del Litoral9.

Junto con su formulación e invitación a resolverlas, siempre sugiero a Alumnos, Pasantes y Adscriptos la conveniencia de construir, en-paralelo o en-simultáneo'10, el diseño de un "proyecto" de investigación aplicada (un pre-diseño o primer esbozo proyectual, en realidad) que les permita por ejemplo:

$>$ transferir o transpolar y recrear principios, dimensiones y categorías analíticas e interpretativas correspondientes a los respectivos dominios del saber, sea que éstos pertenezcan a determinadas orientaciones de la semiótica textual, o de la perspectiva narratológica, o de la práctica analítica del discurso, o del comentario espectacular (en general) y/o cinematográfico (en particular)..., o bien a otros campos (inter)disciplinares afines;

$>$ comprobar o verificar las posibilidades de transponer dichos constructos a ciertas prácticas propias de la indagación cualitativa, con eventuales alcances y derivaciones (por ejemplo) en algunos de los campos de las investigaciones semióticas específicas o aplicadas; o bien en las prácticas de la enseñanza en diferentes especialidades de las ciencias humanas y sociales.

\footnotetext{
${ }^{8}$ Versiones iniciales de estas notas aparecieron también en [2000] "Semiótica general: argumentos, actividades, bibliografía" (CEMED/UNL), en [2001] "Material de Cátedra de Análisis del discurso historiográfico", y en [2002] "Papeles del Seminario sobre Narrativas, Nro 4" (FHUC/UNL).

${ }^{9}$ Me refiero a las Cátedras <Semiótica general>, <Problemática de la literatura y las artes actuales $>$ y $<$ Análisis del discurso historiográfico > , a mi cargo, de las Carreras de Profesorado y Licenciatura en Letras e Historia, y de los Seminarios más recientes de < Investigación semiótica sobre narrativas audiovisuales $>$ y sobre < Semiótica del teatro $>$.

${ }^{10}$ Dicha "construcción" opera, efectivamente (en-paralelo o en-simultáneo) con el (re)descubrimiento de los saberes y conocimientos constituyentes de los respectivos campos y dominios disciplinares involucrados.
} 


\section{REQUERIR CIERTAS RESPUESTAS}

A diferencia de las tareas "re-productivas" que a menudo planteamos en el aula (por caso, la resolución de determinados ejercicios, en los cuales los alumnos "aplican" o "practican" técnicas, destrezas, procedimientos... ya apre[he]ndidos), las actividades relacionadas con la resolución de problemas implican o demandan desarrollar otros procesos de adquisición de aprendizajes y de producción de conocimientos. Esto es: buscar / encontrar respuestas entre aquello que-se-sabe y lo que-se-desea-saber.

A este respecto sugiero considerar y desarrollar las siguientes consignas iniciales:

(1.) Propongan (cotejen o consulten) bibliografía específica de los dominios de la Didáctica y de la Investigación en Ciencias Sociales en que se desarrollen nociones o propuestas afines con esta concepción de <resolución de problemas $>$. De la bibliografía propuesta, seleccionen enunciados que se "orienten" al logro de las mismas expectativas referidas, y discutan sus alcances, implicancias y/o limitaciones ${ }^{11}$.

(2.) Establezcan relaciones posibles entre los resultados obtenidos al resolver la consigna anterior, la estructura conceptual-metodológica de las Cátedras o

\footnotetext{
${ }^{11}$ Propongo a continuación una manera más específica o puntual de resolver la consigna enunciada. Por ejemplo, seleccionar (en primer lugar) en el volumen de los "Contenidos Básicos para la Educación Polimodal" [1ª ed. MCECFCyE, julio 1997] algunos enunciados que, en la formulación de sus contenidos (conceptuales, actitudinales y, fundamentalmente, procedimentales), se "orienten" al logro de aquellas mismas expectativas u objetivos que estamos considerando.

Para el desarrollo de esta actividad sugiero considerar, en especial, el diseño curricular de los siguientes apartados de dicha publicación:

>Los CBC de "Humanidades" (págs. 233-52), "Ciencias Sociales" (135-58), "Tecnología" (159-81) y contenidos "actitudinales generales" (253-7).

> Los CBO y CD de las Modalidades: "Humanidades y Ciencias Sociales" (311-34 [esp. cap.2: 329-34] y 411-24)

y “Comunicación, Artes y Diseño" (359-86 [esp. cap.2: 374-86] y 433-42]

Después de analizar e interpretar dicho repertorio de enunciados, confrontar por ejemplo las conclusiones de dicha actividad con las observaciones (que pudieran derivarse) de una primera lectura de capítulos seleccionados del volumen "Aprender investigando ciencias sociales" de M.Giacobbe y N.Moscoloni [1999: 11-28 y 29- 34].
} 
Seminarios en curso, y las propuestas o sugerencias propedéuticas efectuadas por los respectivos Equipos Docentes.

(3.) Entrando ya en materia específica (la construcción de "su propio" diseño investigativo), le propongo desarrollar las siguientes actividades:

(3.1.) Generar distintas "ideas potenciales" para ser investigadas, desde una perspectiva científica, y en el contexto de un paradigma interpretativo/crítico de investigación.

(3.2.) Seleccionar, en dicho registro o repertorio, aquella "idea" que se considere más productiva (es decir: que aliente el interés o que mejor predisponga para el inicio del trabajo de investigación) y tratar de formularla con la mayor precisión ${ }^{12}$.

\section{INCENTIVAR SUS MOTIVACIONES}

Las siguientes consignas intentan profundizar y expandir los resultados e implicaciones de las actividades propuestas anteriormente:

(4.) Indicar las principales fuentes generadoras de aquella "idea inicial" finalmente seleccionada. Por ejemplo: una experiencia individual, un tema ocasional de conversación o debate, algunos materiales escritos $u$ otras producciones (película, novela, ensayo, etc.), apreciaciones personales u observaciones sobre hechos, ciertas "creencias" y, aun, determinados "presentimientos"... etc., etc. (5.) Elaborar un breve informe acerca de dichas motivaciones, para dejar constancia del origen del proceso y, además, para comprobar que la calidad de la idea (y de la propuesta investigativa subsiguiente) no depende ni se relaciona, necesariamente, con la fuente de donde proviene.

\footnotetext{
12 Un ejemplo posible acerca de cómo enunciar, en su primera versión, la "idea potencial" de un diseño investigativo: "Me propongo estudiar, a través de la emisión de distintos noticieros televisivos (basándome sobre todo en sus segmentos informativos, y también en algunas entrevistas efectuadas a determinados 'candidatos'), los diferentes procesos de mediación que se establecen, en dichos textos audiovisuales, a propósito de las formas de construcción del discurso político".
} 
(6.) Revisar artículos u opiniones ya emitidas a propósito de la misma cuestión (esto es: un primer relevamiento y lectura fragmentaria de otras fuentes documentales o referentes bibliográficos iniciales), a fin de avanzar en la "traducción" del problema en términos cada vez más concretos.

(7.) Determinar, si fuera posible, otras perspectivas de abordaje que en principio pudieran resultar "compatibles" con el enfoque semiótico, discursivo y/o narratológico del tema elegido (por lo menos, según lo privilegia nuestro caso), y que permitan abordar la misma idea de investigación desde diferentes ángulos: sociológico, psicológico, antropológico, comunicológico, didáctico, etc.

\section{PLANTEARSE LOS PROBLEMAS}

Aquella idea inicial habrá de desarrollarse, afinarse, estructurarse más formalmente (incluso modificarse o transformarse), de manera progresiva, a lo largo del proceso de elaboración de su proyecto.

Con esta permanente "(re)constitución del objeto de estudio" tienen que ver:

(1) el planteamiento del problema de la investigación, instancia fundamental en toda pretensión de avance del conocimiento [Bachelard], y

(2) su correspondiente formulación, en términos cada vez más específicos (o en forma tan concreta y explícita como fuera posible, de manera tal que dicho problema sea susceptible de ser investigado a través de procedimientos científicos).

Algunas necesarias consideraciones previas ${ }^{13}$ acerca del planteamiento y formulación de un problema de investigación: enunciar un problema investigativo supone, entre otros aspectos

\footnotetext{
${ }^{13}$ Unas y otras aclaraciones le permitirán ir precisando y delimitando el área-problemática en cuestión. A la vez, le sugerirán actividades pertinentes para iniciar el trabajo investigativo. Aunque, naturalmente, dichas actividades podrán ir siendo modificadas o sustituidas por otras; incluso, incorporadas nuevas variantes a las originales (dependiendo todo ello -claro- de la "dirección" que vaya adoptando la indagación en su transcurso).
} 
$>$ Establecer los objetivos principales que se persiguen.

[En relación con nuestro tema inicial sugerido (ver nota 12) podrían proponerse, por ejemplo, los siguientes objetivos:

- relevar algunos recursos de intervención propios del género 'notitelevisivo', respecto (o a propósito) de la producción de los "textos políticos";

- analizar en particular las modalidades manipulatorias, estratégicas y discursivas de dichas intervenciones;

- focalizar en especial los registros gestuales, quinésicos, proxémicos, paralingüísticos, etc., etc.];

$>$ Desarrollar preguntas direccionales de la investigación.

[En nuestro caso, p.e:

- ¿cómo argumentan los periodistas al narrar la noticia (o al interpelar al entrevistado)?, - ¿de qué manera intervienen los recortes, las elipsis, los "subrayados" (verbales y no verbales) que le imprimen a su "argumentación"?...]; y

> Contextualizar esas referencias, acompañándolas de una breve explicación que

(a) establezca los límites espacio-temporales del estudio [P.e.:

- concentración del interés en la coyuntura electoral de..., en... // entre las emisiones del / los programa/s... // desde... y hasta...], y

(b) esboce un perfil de sus unidades selectivas de observación [Ejs.:

- los mecanismos discursivos de la 'puesta-en-escena' de la entrevista televisiva,

- las relaciones establecidas entre el enunciador político y sus (pro / contra / para) destinatarios...].

\section{COTEJAR OTRAS PROPUESTAS}

Relacionado con unas y otras consideraciones generales (de las recién expuestas), propongo resolver ahora las siguientes consignas 
(8.) Vinculadas con los resultados del estudio realizado ${ }^{14}$ por la investigadora Lucrecia Escudero [cf. (1996): "Malvinas: el gran relato. Fuentes y rumores en la información de guerra". Barcelona: Gedisa. Páginas 35-87]:

(8.1.) Tratar de replantear (en forma resumida) el problema que podría haber motivado oportunamente dicha investigación, según se enuncia o se desprende de la lectura de las páginas indicadas;

(8.2.) Procurar contextualizar dicho problema, haciendo además referencia a sus objetivos iniciales y a sus 'presuntas' preguntas direccionales.

(9.) Respecto de la "idea inicial", anteriormente elegida, para el desarrollo de su propio proyecto de investigación:

(9.1.) Transformarla en el planteamiento de un problema de investigación para "su" proyecto, y elaborar un breve informe que sintetice las cuestiones previas consideradas (objetivos, preguntas, contexto...)

(9.2.) A modo de autoevaluación (de dicho informe), procure responderse las siguientes preguntas: (a) ¿son los objetivos enunciados suficientemente claros, concretos y precisos como para orientar la realización de la investigación?; (b) ino resultan ambiguas, o demasiado globales las interrogantes planteadas?; (c) iexiste suficiente congruencia entre los objetivos y las preguntas de investigación?; (d) ¿está adecuadamente acotado y contextualizado el problema investigativo, en su formulación?; (e) ¿podría mejorarse su enunciación? (en tal caso, ¿de qué manera?; o, directamente, reescribirlo).

\section{FORMULAR EL PROBLEMA}

Avanzar en la formulación del problema de investigación (además de resolver las preguntas iniciales (a) ¿qué se pretende con el trabajo? [objetivos], y (b) ¿qué respuestas deben encontrarse? [interrogantes]), supone también cuestionarse acerca de la justificación de la propuesta, su factibilidad, eventuales

\footnotetext{
${ }^{14}$ Algunas de las siguientes consignas se relacionan con actividades de reflexión y práctica investigativo-bibliográfica aplicadas a la lectura, análisis e interpretación crítica de tres estudios desarrollados por investigadores argentinos del campo de la semiótica y el análisis del discurso: Lucrecia Escudero, Oscar Steimberg y Oscar Traversa.
} 
consecuencias de sus resultados... Indagar, en síntesis, en los siguientes aspectos del problema investigativo:

$>$ Justificar las razones que motivan el estudio propuesto (las cuales ya anticipan índices de impacto del proyecto de investigación resultante); es decir: por qué es conveniente llevar a cabo la investigación y cuáles serían (en principio) los beneficios o utilidades que se derivarán de ella;

$>$ Analizar su 'viabilidad' o factibilidad, en relación con la disponibilidad de recursos (humanos, financieros, materiales, etc.) que determinarán los alcances de la investigación; y

> Cuestionarse acerca de las eventuales consecuencias de la indagación emprendida (por ej: sus implicaciones éticas o estéticas, determinadas afectaciones personales o institucionales, etc).

Se proponen asimismo algunos criterios (flexibles, no exhaustivos) para apreciar el valor potencial de una investigación en curso:

> Conveniencia (en cuanto a sus utilidades o beneficios, por ej.);

$>$ Relevancia (en términos de avance del conocimiento, por ej.: valor teórico, utilidad metodológica...);

> Significación (del problema 'práctico' a cuya solución se orienta, por ej.);

$>$ Transferencias posibles (eventuales usuarios, formas concretas de utilización de los resultados...);

$>$ Otros aportes (por ej., a la formación de recursos humanos, al desarrollo pedagógico o didáctico, etc.)

En relación con las caracterizaciones anteriores, sugiero resolver las siguientes consignas:

(10.) Inferir "criterios" de justificación, de factibilidad y de evaluación de consecuencias de la investigación desarrollada por Lucrecia Escudero [cf. (1996)]. (11.) Relacionar e integrar la consideración de estos nuevos aspectos, a los 
resultados de la tarea ya efectuada (anteriormente, con este mismo estudio), acerca del planteamiento del problema, su contextualización, objetivos e interrogantes.

(12.) Complementar los avances ya efectuados en el diseño de "su propio" proyecto (a propósito de la formulación del problema de la investigación que se quisiera llevar a cabo), con los nuevos elementos de juicio incorporados en esta actividad.

(12.1.) Reelaborar, luego, el anterior informe inicial, condensando el planteamiento del problema (procurar enunciarlo de manera resumida, en no más de tres párrafos -máximo: 300 palabras).

(13.) Establecer un listado de (entre diez y quince) calificativos o factores condicionantes, en virtud de los cuales no podría aceptarse el planteamiento de un problema cualquiera de investigación (por ej.: ambigüedad, excesiva globalidad, desorganización, inconsistencia, etc.).

(13.1.) Confrontar si dichos factores condicionantes inciden (y en tal caso, de qué manera), o no, en el «propio» informe anteriormente elaborado (cf. 12.1). (14.) Teniendo en cuenta alguna tipología de diseños investigativos (por ej. la formulada por Giaccobe-Moscoloni [1999: 18-21])15, ¿con qué tipo(s) de diseño(s) se podría adecuar "su" propuesta de estudio, considerando sólo (al menos en esta fase inicial) el planteamiento del problema de investigación?

\footnotetext{
${ }^{15}$ Como sostienen las autoras, la elección de un determinado tipo de diseño de investigación "depende del estado del conocimiento sobre el tema y el enfoque o marco teórico con que el investigador pretende trabajar" [1999:18]. Caracterizan, en particular, los diseños (1) exploratorios (utilizados para adquirir conocimientos sobre la situación o para generar hipótesis previas a la formulación del problema específico / Ej. Detectar si existen variantes en la construcción del concepto de democracia en los adolescentes pertenecientes a diferentes sectores sociales); (2) descriptivos (centrados en las características del objeto de estudio, para la producción de diagnósticos / Ej. Analizar las características y funcionamiento del subsistema disciplina en el Nivel Polimodal); (3) correlacionales (establecen relaciones entre diversos conceptos o variables / Ej. Reconstruir las prácticas escolares y la significación entre docentes en torno a los fenómenos del poder y la autoridad, en una perspectiva de transformación escolar); (4) transversales (interesados en el análisis de las relaciones entre las variables / Ej. Indagar la relación entre atracción física y confianza durante el noviazgo en las parejas jóvenes, en una ciudad, en cierto momento); (5) longitudinales (estudian las relaciones entre variables a través del tiempo / Ej. Conocer la actitud de una comunidad ante un determinado tema, durante los últimos diez años), explicativos, predictivos, etc.
} 


\section{CONSTRUIR MARCOS REFERENCIALES}

Una vez planteado el "problema" de la investigación y evaluada su relevancia y factibilidad (es decir: no necesariamente 'a posteriori' de dicha actividad, por más que se trate de la opción sugerida para este modo de procesamiento), corresponde sustentar teóricamente el estudio por realizar: elaborar / "construir" el marco teórico del proyecto. Esto es: relevar -recopilar y revisar-, analizar y exponer aquellos enfoques teóricos, antecedentes en general e investigaciones realizadas en particular, que se consideren válidos, confiables y necesarios para un adecuado encuadramiento de la propuesta.

Habitualmente, a partir de un efectivo y (en lo posible) exhaustivo "relevamiento" de dichas fuentes documentarias, suele derivarse la estrategia más conveniente para la construcción del marco referencial de la investigación. "Adoptar una teoría", o "desarrollar una perspectiva teórica" pueden depender, en este sentido, de distintas corroboraciones críticas, las cuales se efectúan a propósito de la existencia previa de:

> Una teoría desarrollada, abundante en "evidencia empírica" y/o capaz de describir, explicar e interpretar fenómenos relacionados con nuestro interés investigativo (aunque hubiera sido motivada por otras inquietudes e interrogantes, o "comprobada" en otros contextos);

$>$ Varias teorías que (habida cuenta de la eventual complejidad de nuestro objeto de estudio, por caso) lo "observan" desde diferentes perspectivas o enfoques, posibles de ser "compatibilizados" entre sí;

> Generalizaciones empíricas, "microteorías" o "fragmentos" de teoría, que sugieren variables potencialmente importantes para la resolución de nuestro problema de investigación; o

> Únicamente guías, aún no investigadas (o exploradas de manera insuficiente), e ideas vagamente relacionadas con el planteamiento de nuestra propuesta, aunque igualmente significativas.

Algunos criterios generales y comunes habitualmente adoptados para evaluar una teoría: (1) capacidad de descripción, explicación, comprensión e interpretación (según los casos: de predicción); (2) consistencia lógica (interrelación, coherencia pro- 
posicional); (3) perspectiva o nivel de generalidad; (4) fructificación (heurística) para generar nuevos interrogantes y descubrimientos; (5) simplicidad y sencillez (obviamente, no equivalentes a "superficialidad"); etc.

Cf. Hernández Sampieri y otros [1997:37-44].

\section{AVANZAR EN LA CUESTIÓN}

Considerando sólo algunas de las muchas complejidades supuestas en toda "construcción de marco teórico" de un proyecto de investigación, propongo reflexionar (a la par que resolver) estas consignas:

(15.) A propósito de la elaboración y exposición de marcos teóricos en tres estudios realizados, respectivamente ${ }^{16}$, por Lucrecia Escudero, Oscar Steimberg y Oscar Traversa:

(15.1.) Seleccionar diversos enunciados que permitan ilustrar diferentes formas de recopilación informativa y sus respectivos modos de relevamiento, referenciación, transposición, etc.

(15.2.) Ejemplificar, en particular, los siguientes casos: (a) resumen de una referencia documental, (b) citación de una idea, concepto, cifra u otro dato, a la que se agrega la opinión del investigador; (c) análisis e interpretación crítica de una referencia documental, efectuada por el investigador; y (d) confrontación de diversas opiniones sobre la referencia, incluida la del investigador.

(15.3.) Procurar reconstituir el "índice" de los respectivos marcos teóricos de investigación, y establecer los criterios de integración u ordenamiento de la información recopilada.

(16.) En cuanto al planteamiento de "su" propia propuesta investigativa:

(16.1.) Seleccionar por lo menos cuatro referencias vinculadas con posibles aspectos de la resolución del problema (extraer de ellas la información perti-

${ }^{16} \mathrm{Cf}$. al respecto: (1) Escudero, L. (1996: 35-87) Malvinas: el gran relato. Fuentes y rumores en la información de guerra. Barcelona: Gedisa. (2) Steimberg, O. (1993: 45-84) Semiótica de los medios masivos. El pasaje a los medios de los géneros populares. Buenos Aires: Atuel. (3) Traversa, O. (1998: 13-36) Cuerpos de papel. Figuraciones del cuerpo en la prensa (1918-1940). Barcelona: Gedisa. 
nente y tratar de exponerla según los modos de recopilación informativa ejemplificados en las consignas anteriores).

(16.2.) Organizar, a partir de dicha selección y elaboración, un posible índice expositivo para el marco teórico de su investigación, y procurar diagramarlo o esquematizarlo.

(17.) A menudo nos encontramos, respecto del término teoría, con definiciones contradictorias o ambiguas. Por otra parte, conceptos como "teoría", "orientación teórica", "marco teórico de referencia", "esquema teórico" o "modelo" a menudo se usan como sinónimos y otras veces con leves matices diferenciales. Al respecto: ¿cuál de las siguientes definiciones de "teoría" ${ }^{17}$ guarda, a su juicio, mayor afinidad con los requerimientos de elaboración del marco teórico de "su" proyecto?

"Una teoría es un conjunto de proposiciones interrelacionadas lógicamente, en la forma de afirmaciones (o de aserciones) empíricas acerca de las propiedades de clases infinitas de eventos o de cosas" [J.Gibbs, 1976].

"Las teorías no sólo consisten en esquemas o tipologías conceptuales, sino que contienen proposiciones semejantes a leyes, que interrelacionan dos o más conceptos o variables al mismo tiempo. Más aún, estas proposiciones deben estar interrelacionadas entre si" [H.M. Blalock, 1980].

"Una teoría es un conjunto de constructos (conceptos), definiciones y proposiciones relacionadas entre sí, que presentan un punto de vista sistemático de fenómenos, especificando relaciones entre variables, con el objeto de explicar y predecir los fenómenos" [F.N.Kerlinger, 1975].

17 Los siguientes conceptos fueron extraidos del manual de Metodología de la investigación de R. Hernández Sampieri y otros autores [1997: 40]. 


\section{ENUNCIAR LAS (PRE)HIPÓTESIS}

La formulación de un problema de investigación nace, en realidad, del imperativo de resolverlo: su razón de ser -podría decirse- está en la búsqueda de una respuesta.

Una vez planteado y contextualizado el problema, mediante la revisión de sus antecedentes, la construcción de su marco teórico..., corresponde establecer guías o explicaciones tentativas (aunque relativamente precisas), a propósito del fenómeno investigado. Esto es, enunciar una o más hipótesis, que nos "indiquen" lo que estamos buscando o tratando de probar.

Las hipótesis suelen ser definidas como (1) proposiciones tentativas (es decir: sujetas a cierto modo de comprobación, que podrán aportar evidencia en su favor o en su contra), (2) acerca de las relaciones existentes entre dos o más variables (o sea: entre "propiedades", cuyos "valores" son susceptibles de algún tipo de "medición"), (3) que se apoyan en conocimientos organizados y sistematizados.

Para su más precisa formulación, las hipótesis debieran ir acompañadas de las correspondientes definiciones "conceptuales" (o dimensiones, componentes de conceptualización teórica) y "operacionales" (o indicadores, niveles observables) de las variables 'contenidas' dentro de cada una de dichas hipóte$\operatorname{sis}^{18}$. Dos precisiones al respecto:

> Por lo común, las hipótesis surgen del planteamiento mismo del problema de investigación: de sus objetivos e interrogantes (ya reevaluados), de los estudios revisados o antecedentes consultados, del postulado de una teoría o de generalizaciones empíricas pertinentes, del análisis de éstas, etc.

$>$ Toda la actividad investigativa (incluida la resolución de sus problemas previos: recolección de datos, de material bibliográfico...) supone la "construcción de hipótesis" (contextualmente verificadas y aceptadas), pero no toda proposición (o afirmación enunciada) constituye una hipótesis.

${ }^{18}$ Para complementar y/o reconceptualizar estas caracterizaciones preliminares sobre la construcción de hipótesis, cf. especialmente, Giacobbe-Moscoloni, ob.cit.: 24-34; H.Mancuso, ob.cit.: 99-158 y H.Sampieri y otros, ob.cit.: 75-106. 
(18.) Relacionar las consideraciones que se efectúan en las siguientes citas ${ }^{19}$ con sus propias experiencias, acumuladas a lo largo de este proceso de construcción de una propuesta investigativa:

"Para que... una proposición conjetural sea considerada o funcione como hipótesis es necesario que los hechos enunciados no hayan sido aún sometidos a la experiencia".

“... para que una conjetura sea una hipótesis se debe admitir (implícitamente, por lo menos), que es corregible, rectificable, modificable o refutable. ...una conjetura es una hipótesis cuando está enunciada en un contexto significativo [sólo en -y a partir de- el cual adquiere 'sentido']".

“... una hipótesis es la explicación de un posible (pero no el único) programa narrativo de un determinado hecho".

Una "hipótesis de lectura [que da sentido a una serie de datos, de otra manera inconexos] es 'una' lectura de lo real, parcial, histórica, complementaria e incluso refutable por otra... Toda hipótesis de lectura es también una hipótesis de comunicabilidad con los otros (por ser social) y un criterio de verosimilitud... de la realidad, mediante la cual tendrán lugar las respectivas 'prácticas históricas' determinadas por ese contexto de reconocimiento".

"Las hipótesis se formulan en un punto dado de la coyuntura cultural y a partir de un conocimiento previo (Peirce). Toda hipótesis... no se formula de modo intuitivo sino mediando un proceso abductivo ${ }^{20}$, crítico y experimental".

\footnotetext{
${ }^{19}$ Las citas corresponden a Mancuso, H. (1999) Metodología de la investigación en ciencias sociales. Lineamientos teóricos y prácticos de semio-epistemología Buenos Aires: Paidós [Cf. cap.3: "Abducción y deducción de hipótesis", págs.99-158].

20 "... la inevitabilidad del 'error' (ya sea entendido como parcialidad o como historicidad) es el horizonte de expectativas de las prácticas humanas. Corolario semioepistemológico: [...] Tal vez sea ésta la definición última del discurso científico: la conjetura (=hipótesis) fundada contextualmente (=socialmente) que se sabe, se presenta, se modeliza, se acepta y se reconoce como permanente conjetura" [H.Mancuso, 1999:157-8, con mis subrayados].
} 
"El proceso de problematización [en el campo de la investigación científica] finaliza... con la formulación de una o más hipótesis que, debidamente contrastadas e incorporadas a un sistema de tesis, se convierten en teorías."

(19.) Procurando situarse en el contexto metateórico de la perspectiva considerada en la consigna anterior:

(19.1.) Releer las propuestas efectuadas (por M.Giacobbe y N. Moscoloni, ob.cit.: 24-34), a propósito de las nociones de unidad de análisis, hipótesis, variables, dimensiones e indicadores ${ }^{21}$.

(19.2.) Ejemplificar dichas configuraciones, valiéndose de los "constructos" (construcciones hipotéticas) ${ }^{22}$ elaborados, para sus respectivas investigaciones, por Lucrecia Escudero, Oscar Steimberg y Oscar Traversa.

(19.3.) Formular una (o más) hipótesis, de acuerdo con el problema que se ha venido planteando, y tratar de definir sus posibles variables en términos "conceptuales" (dimensiones) y "operacionales" (indicadores).

\footnotetext{
${ }^{21}$ Si pretendemos algún grado de confrontación empírica en nuestras hipótesis (=juicio de carácter conjetural) del campo humanístico y social, los conceptos deberán referirse a una cierta "población en estudio" (=unidad de análisis: personas, grupos, instituciones, etc.). Si dichos conceptos teóricos expresan juicios con algún grado de generalización son considerados variables (=propiedades predicadas de las unidades de análisis, las que pueden variar -adquirir diversos valores-y cuya variación es susceptible de medirse) las cuales, a través de un procedimiento de progresiva desagregación en niveles, puede llevarnos a una "definición" de variable efectivamente medible. Las dimensiones de un concepto son los distintos aspectos en que puede ser considerado (=representan las "componentes" de una variable); sus indicadores permiten medirla o cuantificarla, para convertirla en "utilizable" p.e. a los efectos de su manipulación estadística.

Cf. ejemplo propuesto: si la hipótesis establece que "...la complejidad que los estudios de posgrado propician en las IES (instituciones de educación superior brasileras=unidad de análisis) es mucho más significativa que la que puede observarse si se consideran sólo los estudios de grado"... las componentes del dato serían: (a) variable=complejidad de los estudios de grado y posgrado; (b) una sola dimensión de análisis, coincidente con la variable; (c) sus indicadores=los diferentes programas de posgrado por área de conocimiento; y sus "valores" o categorías operacionales: simple, poco compleja, compleja...

22 Las hipótesis y sus variables adquieren "valor" para la investigación científica cuando pueden ser relacionadas con otras (hipótesis / variables) y pasar así a formar parte de una teoría. En este caso se las suele denominar constructos o construcciones hipotéticas.
} 


\section{INTEGRAR APORTES Y AVANCES SUCESIVOS...}

(19.4.) Confrontar y establecer nuevos puntos de contacto y de divergencia entre los alcances de algunas de las anteriores conceptualizaciones (incluidas las definiciones de "teoría" consideradas en las actividades $15^{\mathrm{a}}$ y sgtes, y los avances ya efectuados en la configuración del "constructo hipotético" de su propio proyecto), y los supuestos de las siguientes, cuyas citas fueron extraídas de investigaciones realizadas en el campo educativo ${ }^{23}$ :

"En el pasado, los intentos por entender el concepto de práctica en el campo de la educación siguieron en general el modelo de las ciencias naturales, donde la teorización es considerada diferente del fenómeno que se estudia. Según este punto de vista, una práctica está sujeta a ser en sí misma un objeto no teórico, algo de lo que las teorías tratan, no algo inherentemente teórico. El objetivo de teorizar según el modelo de las ciencias naturales es llegar a tener mayor control técnico sobre el mundo fenoménico [...].

Una hermenéutica de la enseñanza tiene intereses más diversos que su predecesora, que trataba de identificar la esencia racional de la enseñanza... representa un sostenido esfuerzo por encontrar sentido, por descubrir mejores maneras de enseñary, para usar las palabras de Rorty, por 'edificar' encontrando un lenguaje mejor para hablar de la enseñanza y, por lo tanto, mejores maneras de enseñar" [H.McEwan, 1995: 151 y 157].

"Las teorías y los conceptos, los esquemas y las categorías, proporcionan claves con las que observar; pero las claves son sólo indicadores, y se debería ser capaz también de experimentar las cualidades indicadas. En este sentido, el lenguaje teórico... puede realizar una función heurística que haga la búsqueda más eficiente, pero en el refinamiento de las sensibilidades los mismos fenómenos se hacen reales en la experiencia" [E.Eisner, 1990: 267].

\footnotetext{
${ }^{23}$ Las citas corresponden, respectivamente, a: McEwan, H. (1995:236-59) "Las narrativas en el estudio de la docencia", en La narrativa en la enseñanza, el aprendizaje y la investigación [comp]. Buenos Aires: Amorrortu (1998). Eisner, E.W. (1990) El ojo ilustrado. Indagación cualitativa y mejora de la práctica educativa. Buenos Aires: Paidós (1998). Litwin, E.(1997) Las configuraciones didácticas. Una nueva agenda para la enseñanza superior. Buenos Aires: Paidós.
} 
"El sentido de denominar e inscribir esta investigación en una propuesta de colaboración, donde la producción del nuevo conocimiento no tiene su asiento en el escritorio de un investigador, implica una nueva reflexión teórica respecto de las prácticas universitarias, donde se integran la investigación y la producción de conocimientos en la práctica. [...]

A nuestro entender, la investigación didáctica debe contemplar diseños en los que se investigue sobre problemas prácticos, con la intención de mejorar la enseñanza, recuperando la voz de los docentes... al estudiar las interpretaciones del investigador, con el objeto de validar sus análisis" (E.Litwin, 1997: 75 y 143).

\section{... PARA DISEÑAR (FORMALIZAR) "SU" PROYECTO}

Una vez definido el tipo de estudio por realizar y establecida(s) la(s) hipótesis o los primeros lineamientos para llevar a cabo la investigación, debiera concebirse la manera (práctica, concreta) de "responder" a las principales interrogantes planteadas.

Esto implica seleccionar o desarrollar un diseño de investigación apropiado (un plan o estrategia construida para-dar-respuesta a... = diseños experimentales, 'cuasiexperimentales', no experimentales, etc. $)^{24} \mathrm{y}$, a continuación, aplicarlo al contexto particular de "su" estudio.

\footnotetext{
${ }^{24}$ Los diseños específicos (experimentales, no experimentales, etc.) permiten responder las preguntas de investigación planteadas y someter a prueba las hipótesis formuladas.

En su acepción más general (sostienen H.Sampieri y otros [1997:107 ss] una situación experimental consiste en "aplicar un determinado estímulo" a un individuo o grupo de individuos, y "observar el efecto" que provoca dicho estímulo en alguna(s) variable(s) del comportamiento de aquél(los). Esta observación puede realizarse, en condiciones de mayor o menor control, en contextos propios de un estudio de "campo" o de "laboratorio". La investigación no experimental, en cambio, es la que se realiza sin manipular deliberadamente variables: no "construye" acontecimientos sino que "observa" situaciones ya existentes (tal y como se dan en su contexto natural, sin provocación intencional del investigador), para después analizarlas.

El principal objetivo metodológico del estudio experimental es lograr la validez interna (del experimento) para alcanzar después su "validez externa": la posibilidad de generalizar los resultados. Los cuasiexperimentos alcanzan
} 
$[\ldots]$

> En dicha etapa de construcción del pre-diseño (de su primera "versión", o de la elaboración de los tramos iniciales del diseño del proyecto), interrumpimos -momentánea, provisoriamente- este "acompañamiento" del proceso de construcción de "su" proyecto de investigación aplicada, de acuerdo con los propósitos ya enunciados al comienzo de esta secuencia de actividades.

> Obviamente... que resta todavía mucho camino por recorrer, antes de estar en condiciones de cumplimentar los requisitos de presentación (por ejemplo) de un "proyecto" de investigación o de tesis.

Entre otros aspectos sobre los cuales resta reflexionar todavía, se mencionan los siguientes: reelaborar los principales conceptos del fundamento teórico del proyecto e impacto de la investigación en curso; construir el corpus analítico y otros elementos relacionados con cuestiones metodológicas o procedimientos técnicos por emplear; definir el plan de trabajo y establecer el cronograma de las tareas de investigación, etc. Cuestiones (todas) estrechamente vinculadas con la "índole" y "especificidad" de cada propuesta en elaboración...25

$>$ Las actividades sugeridas (seguramente limitadas e insuficientes, como se anticipó en la Presentación de este "Cuaderno") pretendieron sin embargo constituir "andamiajes" para aproximarlo a la compleja y delicada cuestión de las indagaciones científicas. Se ha procurado así (una vez más) "encontrar" correspondencias y "proponerle" algunas formas de interacción posible entre los dominios de la semiótica (sus principales conceptualizaciones, temáticas, referencias teórico-metodológicas...), la enseñanza ('estilo' didáctico, aplicaciones al campo...) y la investigación.

validez interna en la medida en que demuestran la equivalencia inicial de los grupos participantes y su equivalencia en el proceso de experimentación.

Los experimentos "verdaderos" constituyen estudios explicativos; los pre-experimentos básicamente son estudios exploratorios y descriptivos; los cuasi-experimentos son fundamentalmente correlacionales; y las indagaciones noexperimentales suelen dividirse en transeccionales y longitudinales.

${ }^{25}$ Razón por la cual preferimos remitir, para ello, al tratamiento específico que dichos aspectos adquieren en los respectivos "modelos" o "propuestas" de proyectos de investigación que se agregan a continuación, en la segunda parte de este "Cuaderno". 
(20.) Como síntesis y conclusión de las actividades hasta aquí propuestas y las sugeridas (o anticipadas) en estos lineamientos preliminares, le requerimos la producción de un informe técnico acerca de la tarea desarrollada.

Para su presentación, le proponemos completar el siguiente formulario-guía ${ }^{26}$ :

\begin{tabular}{|l|c|}
\hline \multicolumn{2}{|l|}{ Denominación del proyecto } \\
\hline $\begin{array}{l}\text { 0. Cuestiones introductorias } \\
\text { (dilemas, vacancias, incentivos, presupuestos...) }\end{array}$ & $150 / 500$ palabras \\
\hline $\begin{array}{l}\text { 1. Resumen del proyecto } \\
\text { (problema, objetivos, interrogantes...) }\end{array}$ & $150 / 300 \quad " 300 " 150 / 300$ \\
\hline $\begin{array}{l}\text { 2. Situación motivadora } \\
\text { (elección y recorte del tema...) }\end{array}$ \\
\hline \begin{tabular}{l} 
3. Antecedentes sobre el tema \\
\hline $\begin{array}{l}\text { 4. Avances en la fundamentación } \\
\text { (marcos conceptuales, [pre]hipótesis, } \\
\text { referencias bibliográficas consultadas...) }\end{array}$
\end{tabular} \\
\hline $\begin{array}{l}\text { 5. Impacto de la propuesta } \\
\text { (importancia, resultados de significación } \\
\text { esperados, posibles transferencias...) }\end{array}$ & $100 / 200$ \\
\hline $\begin{array}{l}\text { 6. Orientaciones metodológicas } \\
\text { (métodos y técnicas, plan de trabajo...) }\end{array}$ & $200 / 500$ \\
\hline
\end{tabular}

\section{HACIA UNA (META)REFLEXIÓN SOBRE PROCESOS...}

Antes de terminar, quisiera dejar esbozadas sólo algunas referencias acerca de (por lo menos) cuatro cuestiones "abiertas" o propuestas para continuar debatiendo, a propósito de estos planteos iniciales sobre investigación:

${ }^{26}$ En todos los casos, tanto los rubros o ítems indicados, como las extensiones y cantidades de palabras propuestas (para/en el 'esquema' o 'modelo' de presentación) son meramente orientadores, estimativos; de ningún modo excluyentes de otras configuraciones de formulario-guía que pudieran apreciarse como más adecuadas o pertinentes. 
$>$ Los lineamientos metodológicos constituyen, por definición, posibles caminos o recorridos a seguir para alcanzar (a través del proceso de investigación) conocimientos pretendidamente "seguros, confiables y demostrables"; y si esto es así es porque toda opción metodológica entraña a la vez una doble opción, epistemológica y ontológica: la "aceptación" de un determinado concepto de conocimiento y de ciencia, y una determinada "teorización" acerca del conocimiento y sobre la naturaleza de la "realidad" sometida a consideración.

> Si desde una comprensión sincrónica del problema, todo proceso de investigación "culmina" con la presentación de un informe (que recoge las conclusiones y hallazgos más significativos del estudio realizado), desde una perspectiva diacrónica de la cuestión investigativa debiera concebirse como un "proceso continuo" (por momentos inagotable, que siempre insinúa nuevas interrogantes y abre diversas alternativas) y descartarse la idea de que aquel reporte final incluye verdades definitivas que "cierran" el proceso de indagación sobre una determinada realidad.

$>$ La elaboración de dicho informe tampoco implica considerar el estudio como un producto de calidad demostrada, que da por supuesta la credibilidad de sus resultados: tales aspectos sólo se garantizan durante el propio proceso de investigación, con la puesta en discusión de sus criterios, estándares e indicadores específicos de calidad, coherencia, globalidad, etc. (todos ellos asociados con supuestos, tópicos y metodologías del diseño del proyecto), o bien se desvalorizan por eventuales defectos e insuficiencias factuales, formales o conceptuales que llevarían a cuestionar la legitimidad de lo producido.

$>$ De las numerosas opciones implicadas en la tarea del investigador, quien por cierto no se limita sólo a poner en práctica métodos o técnicas sino que permanentemente toma decisiones acerca del "mejor" modo de investigar (grado de compromiso o distancia que asume respecto de la realidad indagada, o de interacción e integración con personas e instituciones vinculadas con su hacer, etc.), se derivan una serie de tensiones y dilemas éticos añadidos a los que, de por sí, ya la afectan: esto es, la presunta neutralidad u objetividad de los procederes inherentes a la investigación poco tiene que ver con la corrección y adecuación de los comportamientos, según "códigos éticos" que debieran regular el desarrollo y la difusión de sus premisas, procesos y resultados. 
La semiótica [dice Roland Barthes en "El susurro del lenguaje" (1984:221-2)] "no es una ciencia como las otras (lo que no le impide ser rigurosa); [...] no quiere en absoluto sustituir a las grandes epistemes, que son algo así como la verdad histórica de nuestro siglo, sino más bien ser su sirvienta: una sirvienta vigilante que a través de la representación de las grandes trampas del Signo, les impide caer en lo que esos grandes saberes nuevos pretenden denunciar: ... ese monstruo que es el Significado Último".

La investigación científica, entendida como proceso (sistemático, dinámico, continuo, "controlado"), constituido por sucesivas etapas interrelacionadas e integradas entre sí, suele orientarse en función de dos propósitos fundamentales: producir conocimientos y teorías (investigación básica) y/o resolver problemas prácticos (investigación aplicada). 